\title{
Une approche de dimensionnement sur cycle de vie pour l'évaluation du potentiel de la distribution DC dans le bâtiment
}

\author{
C. Jaouen ${ }^{1,2}$, B. Multon ${ }^{2}$, F. Barruel ${ }^{1}$ \\ 1. Laboratoire des Systèmes Solaires, CEA-INES, \\ 50, avenue du Lac Léman, F-73377 Le Bourget du Lac, France \\ cedric.jaouen@iut-tarbes.fr; franck.barruel@cea.fr \\ 2. SATIE, ENS Cachan Bretagne, CNRS, UEB \\ av. Robert. Schuman F-35170 Bruz, France \\ bernard.multon@bretagne.ens-cachan.fr
}

\begin{abstract}
RESUME. L'évolution du contexte énergétique associé aux bâtiments (systèmes $P V$ en toiture et véhicules électriques) pose à nouveau la question de la distribution en continu (DC). Dans un contexte où les besoins énergétiques et les impacts environnementaux prennent chaque jour plus d'importance, le dimensionnement d'un tel système de distribution sur sa seule phase d'usage ne répond pas complètement à la question de l'impact global des usages. Autrement dit, le seul fait de réduire le nombre d'étages de conversion électrique et les pertes associées ne permet pas à lui seul d'affirmer que la distribution en DC est plus performante que celle en AC. Pour cette raison, nous proposons une approche d'éco-dimensionnement des composants constituant ce système sur la base d'une minimisation de l'énergie primaire qu'ils consomment pendant tout leur cycle de vie (pertes + énergie grise), l'un des objectifs étant d'évaluer le niveau optimal de tension d'un tel réseau DC. Cet article présente d'abord les modèles élaborés pour le câblage ainsi que pour les convertisseurs électroniques de puissance dans l'optique de déterminer leurs pertes et leur énergie grise. Enfin, des premiers résultats d'optimisation des éco-dimensionnements et des études de sensibilité sont présentés.

ABSTRACT. Evolution of building energetic context (photovoltaic production and electric vehicle) incites us to reconsider the use of DC distribution scheme for building. Since energetic demand and environmental impacts become more and more important, the sizing of such system on its only use phase is nowadays not sufficient to quantify its global environmental impacts. In other words, the only reduction of the number of conversion stages and losses does not in itself evidence that the DC distribution is more efficient than AC. For this reason, we propose an eco-design approach of the components of such system minimizing their primary energy consumption during their whole life cycle (losses and embodied energy). One of the objectives was to evaluate the optimal voltage DC of such network. First, this paper presents the models which have been developed to assess losses and embodied energy of wiring and power electronic components. Finally, some eco-design results and sensitivity studies are presented.
\end{abstract}

European Journal of Electrical Engineering - VOL 16/1 - 2013, pp.87-109. 
MotS-CLES: Distribution en courant continu, Bâtiment, Convertisseur DC/DC flyback, Écoconception, Cycle de vie, Pertes, Energie grise, Energie primaire.

KEYWORDS: DC distribution network, Building, DC/DC flyback converter, Ecodesign, Life cycle, Losses, Embodied Energy, Primary Energy.

DOI:10.3199/JESA.45.1-n (c) Lavoisier 2012

\section{Extended abstract}

Evolution of building energetic context (photovoltaic production and electric vehicle) incites us to reconsider the use of DC distribution scheme for building. Since energetic demand and environmental impacts become more and more important, the sizing of such system on its only use phase is nowadays not sufficient to quantify its global environmental (and economic) impacts. In other words, the only reduction of the conversion stage number and their losses are not sufficient to conclude that the DC distribution is more relevant than AC. For this reason, we propose an eco-sizing approach of the components of such system minimizing their primary energy consumption (gross energy requirement GER) during their whole life cycle (losses and embodied energy). One of the objectives was to evaluate the optimal value of DC voltage of such network.

In the first part of this article, a case study is proposed with a $20 \mathrm{~m}$ length wiring and a flyback DC/DC converter with specific load profile. The eco-sizing approach is also presented. This original demarche is based on the optimization of component size and control parameters to minimize the GER of the wiring and the converter. Then, we present the models which have been developed to assess embodied energy and losses of power electronics components and wiring. In this paper, we especially focus on the models for capacitor and wiring. The other models have already been described in (Jaouen et al 2011a and 2011b). These models are based on the study on some component technologies such as multicellular planar MOSFETs for the switch, Schottky diodes, aluminum electrolytic capacitors and inductor made with two "E core" based on N97 ferrite material. These choices were dictated by the data availability. Then, we present the influence of the DC distribution voltage level on the eco-sizing of a flyback DC/DC converter and wiring supplying a laptop. For these components, we show that higher the voltage level is, smaller the GER is. Moreover we present the contribution of wiring and flyback DC/DC converter inside the GER system and the influence of coupling, between the two components, on the same quantity. Finally, we present the influence of our assumption on expected use duration and on consumption profile on the eco-sizing results before to conclude and give some prospects for further work. 


\section{Introduction}

Le contexte du fort taux de pénétration des systèmes photovoltaïque (PV) en toiture sur les bâtiments et l'arrivée des véhicules électriques, pouvant être acteurs du système électrique (recharges solaires, soutien durant les périodes de pointe, etc...), posent sous un nouveau jour la question de l'évolution de la distribution électrique des bâtiments, actuellement en courant alternatif (AC), vers une distribution en courant continu (DC) (voir Figure 1). Si l'on s'intéresse à l'ensemble des charges présentes dans un bâtiment, elles peuvent être classées en trois groupes :

- les « charges électroniques » fonctionnant intrinsèquement en courant continu (DC) ;

- les charges résistives sensibles à la valeur efficace du courant, capables donc de fonctionner indifféremment en $\mathrm{DC}$ ou en $\mathrm{AC}$;

- les charges électromécaniques qui, si elles sont à vitesse variable (améliorant grandement leur efficacité), exploitent un bus DC.

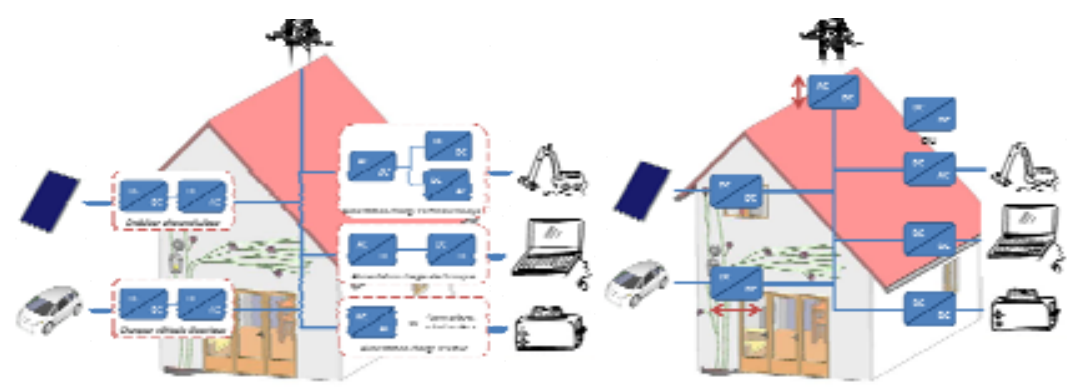

Figure 1 : Architecture actuelle pour la distribution en courant alternatif au sein des bâtiments tertiaire (gauche) et proposition d'une architecture de distribution en courant continu (droite)

Ces charges sont donc d'ores et déjà compatibles avec une distribution en courant continu ou susceptibles de l'être sans modifications techniques majeures. La production en courant continu des panneaux PV et les échanges (éventuellement bidirectionnels) avec la batterie du véhicule électrique posent de façon plus aigüe la question de la pertinence du passage à une distribution en continu au sein des bâtiments. Pour ce faire, le système de distribution DC pourrait être connecté au réseau via un onduleur bidirectionnel, permettant de réinjecter la production $\mathrm{PV}$ lorsqu'elle est en excès ou de prélever de l'énergie au réseau lorsqu'elle est insuffisante. Parmi les différentes questions à résoudre, se pose celle du choix du niveau de tension. Ce choix est bien sûr lié aux aspects sécurité mais également au volet économique. Au sujet de la sécurité, pour les êtres vivants, le courant continu 
est moins dangereux que le courant alternatif à même valeur efficace de la tension (Meyer et Poyard 2006) mais il est plus complexe à gérer du point de vue des protections du matériel à cause de la coupure de l'arc notamment (l'assistance électronique peut constituer alors un intéressant complément). Dans cet article, nous n'étudierons pas le volet protection bien qu'il soit évidemment très important, tout comme les aspects normatifs d'ailleurs.

Plusieurs études ont déjà été menées comparant l'efficacité énergétique d'un même système alimenté en courant continu avec différents niveaux de tension choisis parmi les niveaux de tension communément employés aujourd'hui (Sannino 2003, Engelen, 2006, et Sechilariu, 2007). C'est d'ailleurs dans le domaine des Data Centers (Brush 2012) que la distribution DC semble actuellement s'imposer, avec des niveaux de tension de l'ordre de 400 à $500 \mathrm{~V}$.

Dans un contexte où les besoins énergétiques et les impacts environnementaux prennent chaque jour plus d'importance, la quantification des performances d'un tel système, sur sa seule phase d'usage (pertes énergétiques cumulées), ne répond pas complètement à la question de l'impact global du produit, y compris sur le plan économique. En effet, la classique comparaison des seules pertes en fonctionnement de systèmes dimensionnés, selon des critères pas nécessairement comparables, ne nous semble pas suffisante. Depuis plusieurs années des méthodes d'analyse sur cycle de vie (ACV) se développent et se normalisent. Ces méthodes permettent de quantifier, a postériori, l'effet d'un produit ou d'un service sur l'environnement selon plusieurs impacts normalisés (émissions de gaz à effet de serre, consommation en énergie primaire, rejets dans l'air, l'eau et le sol...) et surtout sur l'ensemble de son cycle de vie (extraction de matières premières, fabrication, distribution, utilisation et recyclage). Elles peuvent donc permettre d'effectuer des comparaisons entre deux systèmes dans le but de choisir celui présentant le plus faible impact environnemental. Dans cet article, nous ne proposons pas de réaliser l'ACV d'un réseau de distribution en courant continu existant, mais nous proposons d'écodimensionner les composants qui le constituent (convertisseurs électroniques et câbles de distribution dans un premier temps), c'est-à-dire de les dimensionner sur des critères environnementaux, ici celui de l'énergie primaire, sur l'ensemble de son cycle de vie. Nous amènerons également des premiers éléments de réponse sur le choix du niveau de tension d'une telle distribution.

En première partie nous présenterons le cadre de l'étude. Nous expliciterons le critère énergétique (Multon et al 2010) (GER : Global Energy Requirement) que nous avons considéré ainsi que la méthodologie employée pour éco-dimensionner le système proposé. Nous présenterons ensuite les modèles utilisés pour quantifier ce critère ainsi que l'approche utilisée pour optimiser le dimensionnement des composants. Enfin nous présenterons l'influence du niveau de tension sur le GER du système en détaillant son influence sur chacun des constituants pris indépendamment. 


\section{Cadre de l'étude et approche}

Nous proposons de considérer un réseau de distribution en courant continu basé sur un unique bus continu (voir Figure 1) sur lequel seraient directement connectées les charges. Dans cette étude d'une grande complexité, nous avons choisi de nous focaliser sur un "échantillon » du réseau de distribution en courant continu d'un bâtiment soit une portion de circuit électrique sur laquelle est connectée une charge de type " électronique " (voir Figure 2). Cette charge est alimentée par un convertisseur DC/DC flyback commandé en mode auto-oscillant, technologie de convertisseur la plus fréquemment utilisée aux faibles puissances et qui nous sert ici de modèle de base pour poser le problème de l'éco-dimensionnement des convertisseurs électroniques de puissance au rôle central dans cette étude. Le niveau de tension du bus continu sera variable afin d'étudier son influence sur le GER du système.

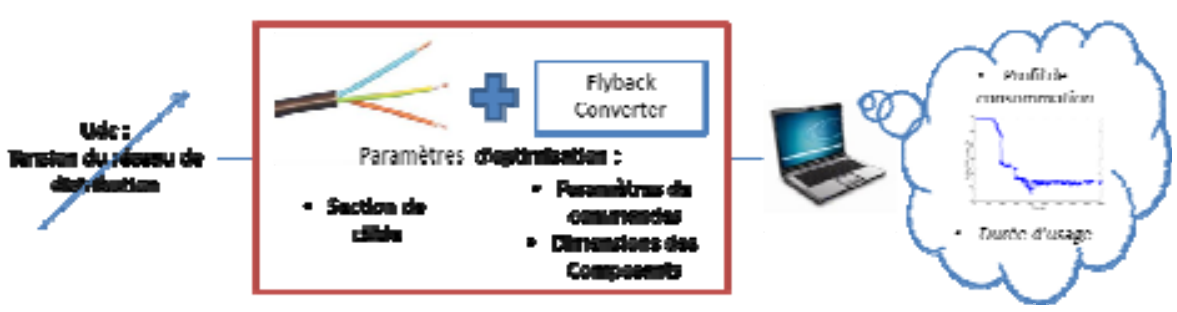

Figure 2 : Cas d'étude considéré (portion de réseau et convertisseur DC-DC)

L'analyse sur cycle de vie est une démarche évaluant une multitude d'impacts environnementaux de nature différente (pouvant donc difficilement être agrégés sous la forme d'un unique critère quantifiant l'impact environnemental d'un système), il nous paraissait donc compliqué de minimiser l'ensemble de ces impacts lors d'une première étape d'éco-dimensionnement. Nous avons donc choisi, dans un premier temps, de ne retenir qu'un seul de ces impacts. Dans le domaine du génie électrique, nous sommes surtout concernés par des dispositifs qui consomment ou produisent de l'énergie et dans lesquels la consommation d'énergie primaire sur l'ensemble du cycle de vie (GER : Global Energy Requirement) revêt une importance significative voire majeure (Multon et al 2010). De plus, une bonne partie des autres impacts est fortement corrélée avec cet impact, c'est notamment le cas des émissions de gaz à effet de serre. Cette consommation englobe :

- les pertes en fonctionnement, évaluées sur cycle, sur toute la durée d'usage (quelques années à quelques décennies selon les parties considérées). Ces pertes sont ramenées en énergie primaire via le rendement du système de production électrique ; 
- l'énergie grise des constituants du système qui correspond à l'énergie primaire nécessaire à l'extraction des matières premières, à la fabrication, mais également à leur transport et leur recyclage.

L'objectif est donc d'optimiser les paramètres de dimensionnement de ce système simplifié en cherchant à minimiser son GER dans l'optique, in fine, de déterminer l'influence de la tension continue du système de distribution sur cette même grandeur. Pour mettre au point la méthodologie de cet éco-dimensionnement, nous avons fait des hypothèses sur :

- les profils de consommation (supposés périodique par soucis de simplification, sachant que la méthode permettra de traiter tout problème plus complexe). Nous considérerons ici un profil de consommation durant $3 \mathrm{~h}$, mesuré sur un chargeur d'ordinateur portable, d'une puissance nominale de 70W (Voir Figure 3). Ce profil est dupliqué une fois par jour, tous les jours pendant toute la durée d'usage ;

- la durée d'usage des constituants. Nous considérerons ici une durée d'usage de 20 ans pour le câble et une durée d'usage de 5 ans pour le convertisseur. On évalue donc le GER du système sur 20 ans en considérant qu'il nous faudra remplacer 3 fois le premier convertisseur pour couvrir cette période. On distingue bien ici la notion de durée de vie, relative au vieillissement des composants, et la durée d'usage, relative au remplacement du matériel pour d'autres raisons (normes, consumérisme...) ;

- la topologie du câblage. Nous considérerons ici arbitrairement, à titre de premier exemple, un câble de 20 mètres de longueur.

Il est important de noter que les pertes en fonctionnement des composants électriques sont fortement liées à la quantité de matière active qui les constitue, ce qui est bien connu dans les dispositifs électrotechniques (Hadj Amor et al 1998). En outre, en première approximation, l'énergie primaire nécessaire à la fabrication des composants du système (énergie grise) croît avec la quantité des matières actives utilisées. Partant de ces constatations, il parait assez simple, dans le cas d'un câble électrique, de choisir la section de conducteur comme paramètre d'optimisation, sachant que la quantité d'isolant lui sera directement liée. Pour chaque niveau de tension, nous déterminerons donc la section de conducteur minimisant le GER du câble, sur le cycle de vie considéré, à l'image de l'étude présentée dans (Jaouen et al 2011a).

En ce qui concerne le convertisseur flyback, nous avons considéré qu'il était nécessaire de détailler son dimensionnement à l'échelle de ses principaux composants de puissance, en négligeant la contribution (au GER du convertisseur) de la commande, de l'écrêteur, du circuit imprimé, de l'éventuel dissipateur et du boitier. Pour limiter l'ampleur du problème, nous nous sommes limités à des technologies particulières (MOSFET multicellulaire planar classique, diode Schottky, noyau magnétique de forme donnée, matériau ferrite N97 Epcos, condensateurs électrochimiques aluminium Vishay à électrolyte liquide). Après avoir dimensionné des composants de référence, nous avons modifié leurs 
dimensions caractéristiques avec des facteurs homothétiques, que nous avons optimisés afin de minimiser le GER (voir Figure 3). Pour les condensateurs et le noyau du composant magnétique, nous avons appliqué un facteur homothétique sur leur volume. Pour la diode et transistor, les substrats étant, dans la gamme de tension considérée, de même épaisseur, seule la surface de puce influe sur les pertes. Nous avons donc appliqué un facteur homothétique sur leur surface.
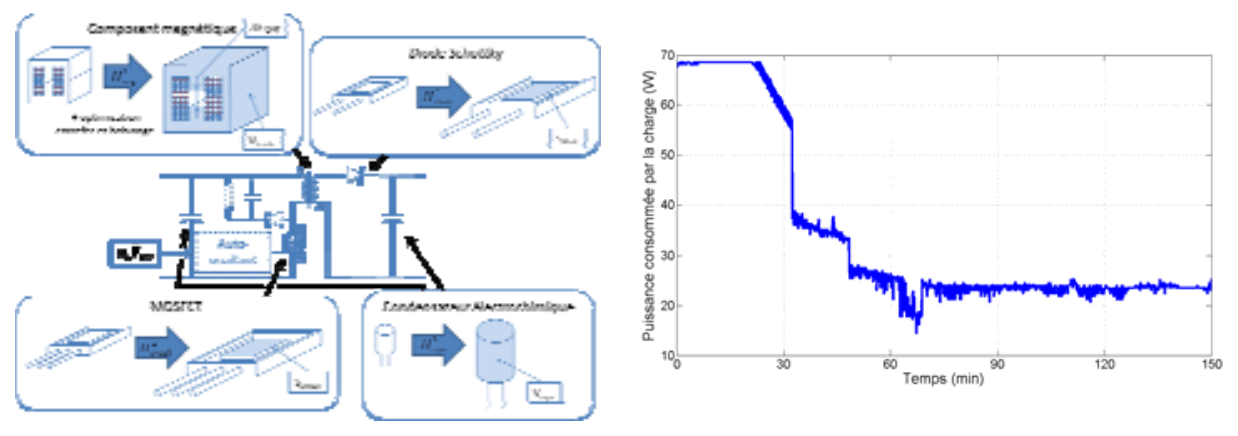

Figure 3 : Approche homothétique appliquée aux composants de puissance du convertisseur DC-DC flyback (gauche) et profil temporel de puissance consommée par la charge sur un cycle type (droite)

Comme le montre la Figure 4, on prédimensionne chacun des composants, pour un niveau de tension et pour un jeu de paramètres de commande donné $\left(\mathrm{F}_{\mathrm{SW}}\right.$ fréquence nominale, $\alpha$ rapport cyclique), un composant de référence sur lequel on appliquera ensuite un facteur homothétique à optimiser. Cette étape correspond à un dimensionnement initial rapide fondé sur le seul respect des contraintes thermiques et électriques au point nominal de fonctionnement. Cependant contrairement au cas du câblage, on est ici confronté à une dépendance des pertes dans le convertisseur vis-à-vis de ses paramètres de commande (fréquence nominale, rapport cyclique) qui constituent donc des paramètres secondaires nécessitant également une optimisation. Il y a donc, comme le montre la Figure 4, deux optimisations imbriquées. Nous déterminons donc pour chaque niveau de tension, les paramètres de commande minimisant le GER du convertisseur (boucle en pointillés) et pour chaque couple de paramètres $\left(\mathrm{F}_{\mathrm{SW}}, \alpha\right)$ nous déterminons, pour chacun des composants, le facteur homothétique minimisant son GER établi pour un cycle de fonctionnement et une durée d'usage fixés (boucle en traits pleins). Pour le composant magnétique, en plus de son facteur homothétique, il est nécessaire d'optimiser les valeurs de son entrefer (et par conséquent les nombres de spires, directement liés à la valeur de l'entrefer) afin de minimiser les pertes en fonctionnement, tout en respectant une contrainte d'induction maximale. L'ensemble de ces optimisations ont été réalisées en utilisant les algorithmes d'optimisation implantés dans le logiciel Matlab ${ }^{\odot}$ permettant de résoudre les problèmes d'optimisation sous contraintes. Le découplage des 
optimisations de chacun des composants est rendu possible en considérant le mode de commande auto-oscillant et en négligeant l'influence des chutes de tension et des pertes d'un composant sur l'autre. L'effet de ces couplages est du second ordre, tant que les chutes de tension et les pertes sont relativement faibles, ce qui aura tendance à être le cas dans un éco-dimensionnement sur de longues durées de fonctionnement. Dans ce dernier cas, il serait alors nécessaire de considérer les couplages au prix d'un alourdissement sensible de la résolution.

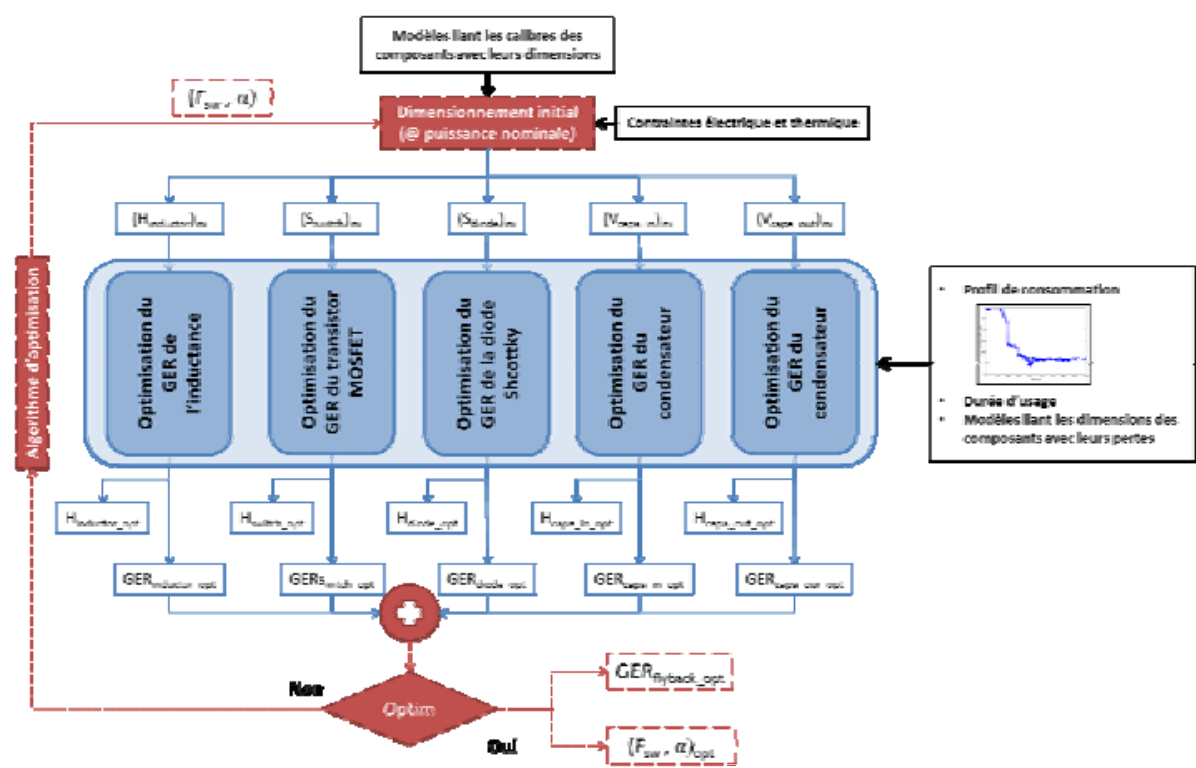

Figure 4 : Synoptique de l'optimisation du dimensionnement des composants de puissance du convertisseur flyback

\section{Modélisation du GER du système étudié}

Dans cette partie nous allons détailler les modèles utilisés pour évaluer le GER du convertisseur DC-DC flyback (en mode auto-oscillant) précédemment décrit et de la portion de câble mentionnée $(20 \mathrm{~m})$. Ils ont pour objectif :

- d'établir, pour chaque composant, des lois, éventuellement empiriques, liant leurs caractéristiques à leurs facteurs dimensionnels et aux paramètres associés à leurs pertes ;

- d'établir les modèles de pertes relativement aux paramètres de dimensionnement ;

- et de lier leurs facteurs dimensionnels à leur énergie grise. 


\subsection{Modélisation du GER d'un câble de distribution (Jaouen et al 2011)}

Comme nous l'avons vu plus haut, le GER est composé des pertes énergétiques en fonctionnement $\mathrm{E}_{\text {pertes }}$ (énergie obtenue par intégration de la puissance dissipée et convertie en énergie primaire via le rendement de production et distribution d'électricité) sur l'ensemble du cycle de fonctionnement caractérisé par une durée d'usage ainsi que de l'énergie grise totale des éléments considérés, sachant que l'énergie «utile» distribuée par le câble est une contrainte liée à l'application et qu'elle n'est donc pas comptabilisée dans le GER.

Les pertes énergétiques par effet Joule sont évaluées à l'aide de l'équation (1), en prenant en compte l'influence de la température $\theta_{\text {cuivre }}$, déterminée en utilisant un modèle thermique statique, sur la résistivité du cuivre (les régimes transitoires ne sont pas considérés à ce stade de l'étude, d'autant plus qu'ils complexifient sévèrement la méthodologie (Debusschere 2009) en nécessitant un traitement temporel),. Dans cette expression $\mathrm{P}_{\text {load }}$ représente la puissance instantanée consommée par la charge, $U_{\mathrm{dc}}$ la tension du bus continu et $\eta_{\text {élec }}$ le rendement de conversion entre énergie primaire et énergie électrique dans le cas du mix énergétique français (Dones et al 2007).

$$
\begin{gathered}
E_{\text {pertes }}=\frac{1}{\eta_{\text {elec }}} \times \int_{\text {Duréed'usage }} \frac{\rho\left(\theta_{\text {cuivre }}\left(P_{\text {load }}\right)\right) \times L_{\text {câble }}}{S_{\text {Cu }}} \times\left(\frac{P_{\text {load }}}{U_{d c}}\right)^{2} d t \\
\text { avec } \quad \eta_{\text {elec }}=0.28
\end{gathered}
$$

Afin d'estimer l'énergie grise du câblage, $\mathrm{EG}_{\text {câble, nous avions besoin de }}$ connaitre le lien entre la section du conducteur et la quantité d'isolant. Nous nous sommes basés pour cela sur la norme française NF C 32-201 qui donne l'épaisseur minimale d'isolant en fonction de la section du conducteur pour une tension allant jusqu'à 450V (voir Figure 5) (NF 1998). Il nous fallait ensuite des données issues d'études ACV estimant l'énergie grise d'un câble. Par manque de données ACV nous avons considéré que l'isolant du câble était constitué de PVC. Nous nous sommes basés sur deux sources, l'une pour le cuivre, l'autre pour le PVC :

- Tikana et al (2005) qui ont évalué à $112 \mathrm{Wh}_{\mathrm{p}}$ l'énergie primaire nécessaire à l'extraction des matières premières, au raffinage, à la fabrication et au recyclage d'un fil de cuivre nu d'un mètre et d' $1 \mathrm{~mm}^{2}$ de section, soit une valeur $\mathrm{EG}_{\text {fil_cuivre }}$ de $112 \mathrm{MWh}_{\mathrm{p}} / \mathrm{m}^{3}$.

- Hischier (2007a) qui a estimé une valeur $\mathrm{EG}_{\text {isolant }}$ de $26 \mathrm{MWh}_{\mathrm{p}}$ d'énergie primaire pour produire un $\mathrm{m}^{3}$ de $\mathrm{PVC}$, de l'extraction des matières premières à la synthèse du matériau.

L'énergie grise du câble sera évaluée, en exploitant ces données numériques, à partir de l'expression (2) avec $S_{C u}$ et $S_{\text {isolant }}$ les sections respectives de l'âme et de l'isolant du câble et $\mathrm{L}_{\text {câble }}$ sa longueur. On cherchera ensuite à minimiser, pour une topologie de distribution, pour une puissance consommée par la charge et pour une durée d'usage données, le GER du câble. Pour ce faire, on déterminera, lors d'un 
dimensionnement initial, la section minimale respectant à la fois les contraintes thermiques $\left(70^{\circ} \mathrm{C} \max \right)$ et les contraintes de chute de tension (5\% max). On fera ensuite varier la section du conducteur et on cherchera celle minimisant le GER somme de l'énergie grise et des pertes du câble.

$$
E G_{\text {cable }}=\left(E G_{\text {fil_cuivre }} \times S_{C u}+E G_{\text {isolant }} \times S_{\text {isolant }}\left(S_{C u}\right)\right) \times L_{\text {cable }}
$$

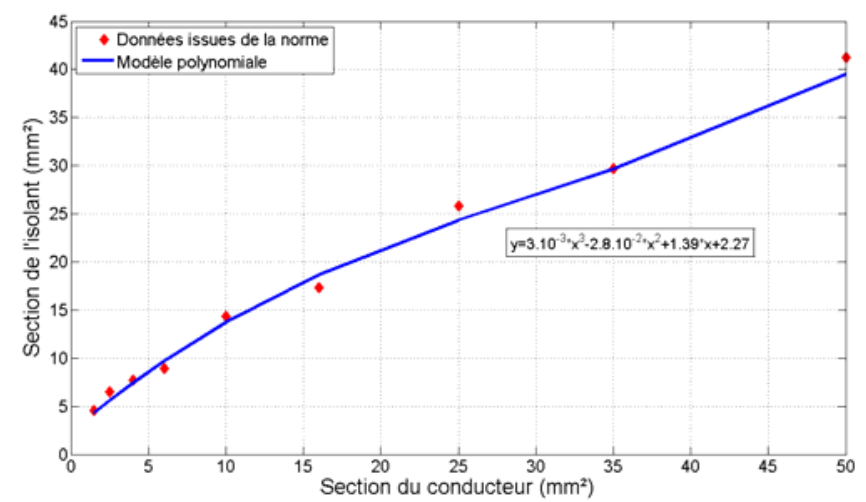

Figure 5 : Modèle polynomial liant la section de l'isolant avec la section du conducteur selon la norme NF C 32-201

On trouvera plus de détails sur l'éco-dimensionnement des câbles dans la référence (Jaouen et al 2011a) traitant de l'éco-dimensionnement d'un câble alimentant un véhicule électrique, ou d'un réseau de distribution pour un système d'éclairage.

\subsection{Modélisation simplifiée du convertisseur DC-DC flyback auto-oscillant}

On présente ici les principes nous permettant de déterminer le GER des principaux composants de puissance du convertisseur flyback. Le régime autooscillant est par définition à fréquence variable. C'est à la puissance "nominale » (maximale) que le régime est le plus contraignant et nous appelons fréquence nominale, la fréquence correspondante (la plus basse), elle sera l'un des paramètres d'optimisation. Le fait que les chutes de tension soient négligées en première approximation conduit à ce que le rapport cyclique soit constant, quel que soit le point de fonctionnement, puisque les tensions d'entrée et de sortie sont considérées constantes. Il constituera également l'un des paramètres d'optimisation. Nous allons maintenant détailler les modèles utilisés pour évaluer le GER des condensateurs de filtrage afin d'illustrer la démarche de dimensionnement. Pour les autres composants on trouvera, en plus des quelques éléments présentés ici, plus de détails dans (Jaouen et al 2011b). Un condensé des modèles utilisés par la suite est donné dans le Tableau 1. 


\subsubsection{GER des condensateurs de filtrage}

A tenue en tension donnée, nous avons choisi le condensateur de référence afin de limiter l'ondulation de tension (en entrée et en sortie) à 10\% (cahier des charges arbitraire). Pour cela nous avons négligé l'ondulation de tension capacitive étant donnée l'effet dominant de la résistance série ESR, pour la technologie considérée (électrolytique) et aux fréquences où travaille le convertisseur (de quelques dizaines à quelques centaines de $\mathrm{kHz}$ ). Le condensateur de référence est donc largement caractérisé par sa valeur d'ESR, supposée constante sur toute la plage de fréquence balayée en mode auto-oscillant.

Avant d'appliquer le facteur homothétique sur les dimensions du condensateur nous avons besoin d'un modèle liant ses dimensions (et ici plus particulièrement son volume, puisque l'homothétie est globale) avec son ESR. Comme on peut le voir sur la Figure 6, l'ESR d'un condensateur se révèle, après observation, globalement inversement proportionnelle à son volume et à un facteur près dépendant de la tenue en tension du condensateur.
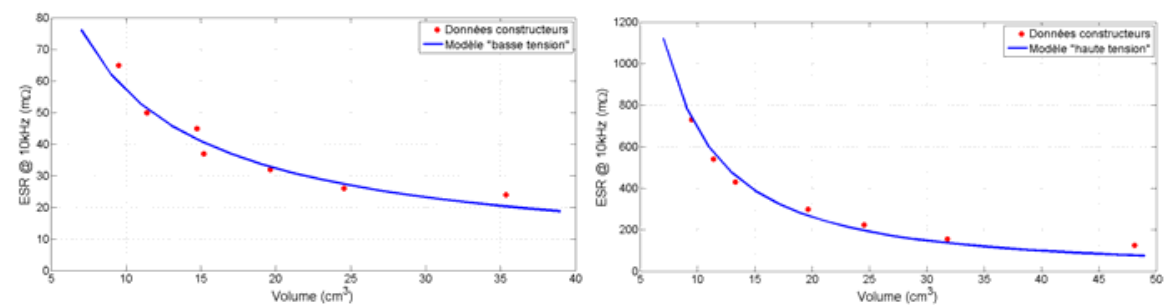

Figure 6 : Résistance équivalente série en fonction du volume pour une tenue en tension de 40V (gauche) et 200V (droite) pour les condensateurs électrochimiques fabriqué par Vishay (série 058/059 PLL-SI).

Nous avons donc développé des modèles reliant l'ESR d'un condensateur avec son volume et sa tenue en tension. Ces lois ont été établies à partir de l'analyse d'une large gamme de condensateurs électrochimiques aluminium à électrolyte liquide fabriqué par Vishay (série 058/059 PLL-SI) (Vishay 2010a). Les contraintes de tenue en tension n'étant pas les mêmes en entrée et en sortie du convertisseur $(<50 \mathrm{~V}$ en sortie et $>50 \mathrm{~V}$ en entrée), nous avons développé un modèle pour les condensateurs « basse » tension et un autre pour les condensateurs « haute » tension. On en donne les expressions selon la gamme de tensions dans l'équation (3) avec $U_{r}$ la tenue en tension des condensateurs et Vol leur volume (exprimée en mm3). La première a été établie à partir de 42 références et pour des tenues en tension allant de 6,3 à $63 \mathrm{~V}$ (erreur moyenne de $+/-8 \%$ ). La seconde provient de l'étude de 28 références et convient pour des condensateurs dont la tenue en tension est comprise entre 100 et 400V (erreur moyenne de $+/-20 \%$ ).

$$
E S R_{\leq 63 V}=74 \times U_{r}^{0.1} \times V^{-0.8} \quad E S R_{\geq 100 V}=370 \times U_{r}^{1.2} \times V^{-1.4}
$$


Une fois le facteur homothétique appliqué sur les dimensions du condensateur de référence nous utilisons également les lois de l'équation (3) pour déterminer la valeur de l'ESR du nouveau condensateur et pour évaluer ses pertes en fonctionnement pour un profil de référence et pour une durée d'usage fixés. Les pertes en fonctionnement dans les condensateurs de filtrage seront calculées en se basant sur la valeur de sa résistance équivalente série (ESR).

Concernant l'évaluation de l'énergie grise d'un condensateur, nous nous sommes basés sur une donnée provenant de l'université de Chalmers (2000). Leur étude a révélé que la fabrication d'un $\mathrm{kg}$ de condensateur électrochimique de hauteur inférieure à $2 \mathrm{~cm}$ nécessite $260 \mathrm{kWh}_{\mathrm{p}}$ d'énergie primaire, de l'extraction des matières premières à la fabrication du condensateur. Pour déterminer la masse d'un condensateur, à volume donné, nous avons, grâce aux données constructeurs, pu déterminer une masse volumique moyenne, $\rho_{\text {capa }}$, de $1400 \mathrm{~kg} / \mathrm{m} 3$. On obtiendra donc l'énergie grise du condensateur, $\mathrm{EG}_{\text {capa, }}$ grâce à l'équation (4).

$$
E G_{\text {capa }}=E G_{\text {massique_capa }} \times V \operatorname{Vol}_{\text {capa }} \times \rho_{\text {capa }}
$$

\subsubsection{GER du MOSFET}

A tenue en tension donnée, le transistor de référence sera celui acceptant thermiquement (dans des conditions d'échange thermique spécifiées par le fabricant) le courant efficace maximal requis par l'application. C'est sur la surface de puce de ce transistor de référence que nous appliquerons un facteur homothétique. Nous avons donc, dans un premier temps, créé un modèle reliant la surface de puce avec le calibre en courant.

Les modèles de calcul de pertes dans les transistors sont fondés sur la résistance à l'état passant $\mathrm{R}_{\mathrm{DSon}}$ (détermination des pertes de conduction) et la charge totale de grille $\mathrm{Qg}_{\mathrm{g}}$ (John Shen et al 2006) pour la détermination des pertes de commutation. Afin de déterminer des lois reliant la surface de puce, notée $\mathrm{S}_{\mathrm{Si}}$, avec ces deux grandeurs et reliant la surface de puce avec le calibre en courant, nous avons analysé des datasheet d'un constructeur de puces nues en technologie multicellulaire planar (IXYS). Nous avons pu en extraire des lois reliant le FOM (Figure Of Merit) (Ejury 2003) et la résistance spécifique $\mathrm{R}_{\mathrm{DSon}} . \mathrm{S}_{\mathrm{Si}}$ avec la tenue en tension du transistor BVDS (voir Tableau 1). Avec ces modèles, et dans des conditions particulières de commande de grille (Jaouen et al. EPE 2011), nous pouvons déterminer les éléments caractéristiques des pertes pour évaluer les pertes en fonctionnement au sein de l'interrupteur.

Pour évaluer l'énergie grise, nous avons exploité une donnée issue de (EIME 2009) qui estime à $512 \mathrm{Wh}_{\mathrm{p}}$ la quantité d'énergie primaire nécessaire pour produire un transistor en boitier TO220, donnée recoupée par des informations collectées auprès d'un fabricant de MOSFET et dont la valeur est très largement due à la phase d'élaboration de la puce. Pour déterminer l'énergie grise d'un transistor on comparera sa surface de puce avec la surface maximale pouvant être empaquetée dans un boitier TO220. Le ratio entre ces deux surfaces nous permettra de calculer 
l'énergie grise du transistor par une simple règle de trois, en supposant donc que les tailles de puce et de boîtier varient continûment.

\subsubsection{GER du composant magnétique}

Le noyau ferrite support est un circuit de type EE10/5.5/5 sur lequel nous appliquons un facteur homothétique (notons que ce choix conduit à une répartition figée de la quantité de cuivre et de matériau ferrite). Le circuit magnétique de référence sera le circuit magnétique respectant la contrainte d'induction maximale $\left(B_{\max }=300 \mathrm{mT}\right)$ et respectant les contraintes thermiques du bobinage et du noyau ferrite $\left(\theta_{\text {Cu_max }}=120^{\circ} \mathrm{C} ; \theta_{\text {ferrite } \max }=100^{\circ} \mathrm{C}\right)$ calculé en statique comme dans (Debusschere 2009) et en omettant les échanges entre le cuivre et le circuit magnétique. Il sera caractérisé par un facteur homothétique de référence $\left(\mathrm{H}_{\text {inductor_ini }}\right)$ à appliquer sur le circuit magnétique support EE10/5.5/5 pour obtenir le circuit de référence.

Pour modéliser les pertes fer volumiques en fonction de la fréquence, du rapport cyclique maximal et de l'amplitude de l'induction, nous avons utilisé le modèle DNSE (Van den Bossche 2005) (Double Natural Steinmetz Equation).

Les données support pour l'évaluation de l'énergie grise ont été extraites de (Hischier et al 2007b) pour le matériau ferrite de $\left(8 \mathrm{kWh}_{\mathrm{p}} / \mathrm{kg}\right)$ et de (Boughamni et al 2010) pour le processus d'émaillage du fil de cuivre $(3.6 \mathrm{kWh} / \mathrm{kg}$ de cuivre). Pour l'âme du bobinage, nous avons considéré la même donnée que celle du câble. Pour chaque facteur homothétique appliqué sur le composant magnétique nous minimisons les pertes en fonctionnement, en optimisant la longueur de l'entrefer ainsi que les nombres de spires (à facteur de remplissage constant).

\subsubsection{GER de la diode Schottky}

La diode de référence sera choisie par rapport à son calibre en courant (valeur moyenne du courant, donnée fabricant) et on appliquera ensuite un facteur homothétique sur la surface de puce. Nous avons donc, dans un premier temps, créé un modèle reliant la surface de puce avec le calibre en courant (voir Tableau 1).

Pour la diode, nous avons émis l'hypothèse d'un seuil de tension indépendant de sa tenue en tension (celle-ci dépend très peu de la tension d'entrée puisque la tension de sortie est constante), et d'une résistance dynamique dépendante de son calibre en courant. Nous avons également négligé les pertes de commutation de la diode Schottky (sans charge stockée et avec un blocage à faible di/dt dû au mode autooscillant). Le modèle liant la résistance dynamique d'une diode Schottky avec son calibre en courant a été élaboré sur la base des documentations constructeurs de la société Vishay (2010b).

N'ayant pas d'autres données que celles du transistor MOS pour les semiconducteurs, nous avons calculé l'énergie grise de la diode de la même façon que pour le transistor, sachant qu'elle est probablement surestimée (moins d'étapes de masquage). 


\section{Influence du niveau de tension sur le GER du système}

Avant de s'intéresser à l'impact du niveau de tension sur le système global, nous allons regarder son influence sur chacun des deux constituants (câble et convertisseur DC/DC).

\subsection{Influence du niveau de tension sur le GER d'un câble d'alimentation}

Nous avions déjà étudié dans (Jaouen et al 2011a), l'impact du niveau de tension sur le GER d'un câble d'alimentation, on rappelle ici les principaux résultats. Pour chaque niveau de tension, la section du câble est optimisée afin de minimiser son propre GER. On présente Figure 7 l'évolution du GER et de la section optimale du câble, soumis au profil de la Figure 3, en fonction du niveau de tension (au premier ordre, on a négligé l'impact des pertes du convertisseur sur les profils).

Comme on pouvait s'en douter, le GER du câble diminue lorsque le niveau de tension du bus continu augmente. En effet, à l'optimum, la somme des pertes énergétiques par effet Joule et de l'énergie grise diminue lorsque la tension augmente. De plus nous avions déjà illustré dans (Jaouen et al 2011a) que pour la section de câble minimisant le GER, il y avait sensiblement égalité entre pertes en fonctionnement et énergie grise (si la masse d'isolant était proportionnelle à la masse de cuivre et pour des contraintes non atteintes, on obtiendrait une stricte égalité).
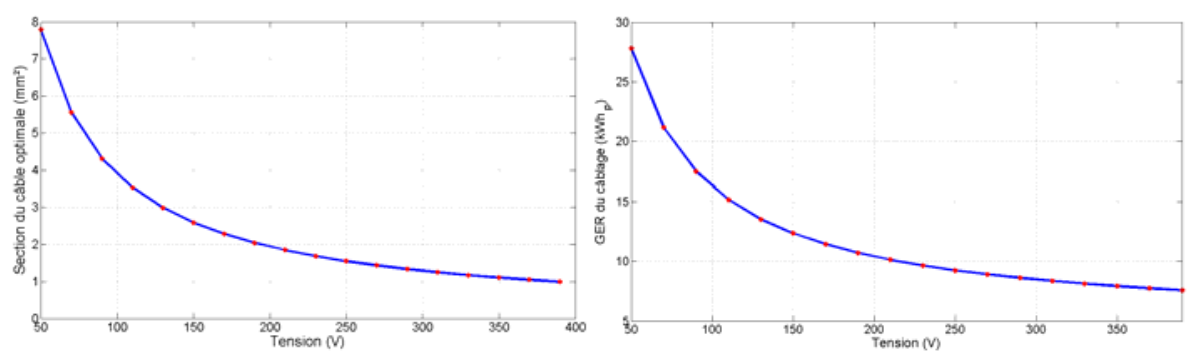

Figure 7 : Evolution de la section de câble optimale (gauche) et Evolution du GER optimal associé (droite) en fonction de la tension du bus continu

Il est aussi important de noter que la section optimale reste valable quelle que soit la longueur du câble puisque les pertes en fonctionnement et l'énergie grise sont directement proportionnelles à la longueur du câble.

\subsection{Influence du niveau de tension sur le GER du convertisseur DC-DC flyback}

On s'intéresse maintenant à l'évolution du GER du convertisseur flyback en fonction de la tension d'entrée (résultats présentés Figure 8). On rappelle que pour chaque niveau de tension, les paramètres de commande ( $\alpha$ et Fsw) sont optimisés de 
façon à minimiser le GER du convertisseur. De même, pour chaque couple de paramètres de commande, les facteurs homothétiques de chaque composant sont optimisés indépendamment de façon à minimiser leur GER.
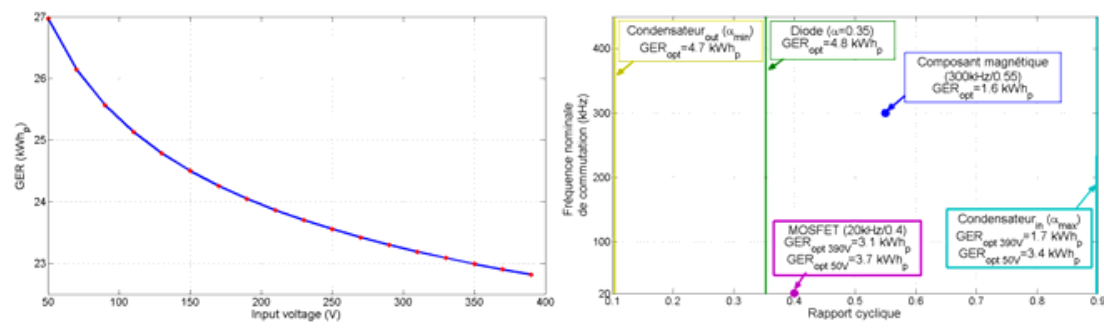

Figure 8 : Evolution du GER du convertisseur en fonction de la tension du bus continu (gauche) et Lieu des paramètres de commandes optimaux associés à chacun des composants et GER associés pour $50 \mathrm{~V}$ et $390 \mathrm{~V}$ (droite).

On peut remarquer que le GER optimal du convertisseur diminue également avec le niveau de tension. Pour expliquer ce phénomène on s'intéresse à l'emplacement des paramètres de commande optimaux pour chacun des composants pris indépendamment (voir Figure 8). Sur cette figure on représente le lieu des minima et le GER associé pour 50 et $390 \mathrm{~V}$. On peut remarquer, dans un premier temps, que les valeurs optimales des paramètres de commandes ne sont pas affectées par l'évolution du niveau de tension. Par contre, seules les valeurs optimales du GER du MOSFET et du condensateur d'entrée diminuent avec l'augmentation du niveau de tension. Pour le condensateur et la diode, le lieu est une droite verticale car la fréquence n'intervient pas. Leur emplacement en sortie du convertisseur les rend également indépendants (sauf pour le condensateur d'entrée) de la tension d'entrée, à rapport cyclique constant. L'algorithme d'optimisation cherche donc à diminuer le rapport cyclique et à augmenter la fréquence du convertisseur afin de diminuer la contribution des composants qui n'ont pas été affectés par le changement de niveau de tension. Le GER du convertisseur va ainsi globalement diminuer avec l'augmentation du niveau de tension.

4.3 Influence du niveau de tension sur le GER du système complet et du couplage entre les composants

Nous allons maintenant nous intéresser à l'évolution du GER du système « câble + convertisseur ». Le fait que les deux « sous-systèmes » pris séparément voient leur GER diminuer avec l'augmentation du niveau de tension d'entrée entraîne, de façon évidente, que le GER du système présentera la même tendance. On présente, Figure 9, l'évolution du GER du système en prenant en compte deux cas de figure :

- dans le premier cas, on additionnera le GER du câble (de $20 \mathrm{~m}$ aller), soumis au profil de consommation de la charge (sans les pertes du 
convertisseur) et le GER du convertisseur alimenté directement par le niveau de tension référence du bus (sans tenir compte des chutes de tension) ;

- dans le deuxième cas, le GER du système est constitué de la somme du GER du câble, en ajoutant au profil de consommation les pertes du convertisseur, et du GER du convertisseur optimisé sous une tension plus faible prenant en compte les chutes de tension dans le câble d'alimentation.
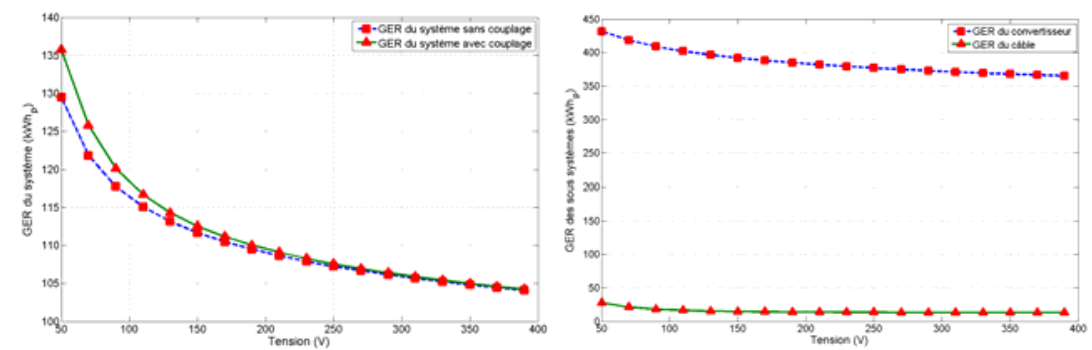

Figure 9 : Effets des couplages (à travers les pertes) entre les optimisations du câble et du convertisseur en fonction du niveau de tension (gauche) et Répartition du GER du système entre le câble et le convertisseur (droite)

On remarque que le couplage est faible entre les deux composants, et ce quel que soit le niveau de tension, puisque le GER du système en prenant en compte les couplages est ici très proche de celui les négligeant. En effet, l'écodimensionnement des convertisseurs conduit généralement à des composants plus gros et donc à de meilleurs performances (rendements élevés et faibles chutes de tension), minimisant les éventuels couplages entre les différents composants. On pourra donc considérer par la suite, dans le cas où les chutes de tension au sein du réseau sont relativement faibles (inférieur au \%), que le système optimal d'un point de vue du GER peut être déterminé en optimisant indépendamment les différents composants.

Si l'on s'intéresse maintenant à la répartition au sein du GER, entre le câble et le convertisseur, on peut remarquer que le câble représente dans cet exemple particulier moins de $15 \%$ du GER du système. Cette proportion est en pratique évidemment liée à la longueur de câble considérée, puisque nous avions déjà illustré dans (Jaouen et al 2011a) que le GER du câble est directement proportionnel à sa longueur, mais également aux considérations de mutualisation de portion de câble, pour alimenter différentes charges, sur le réseau DC. 


\section{Etude de sensibilité}

L'éco-dimensionnement que nous venons de présenter a été réalisé avec un profil de consommation particulier et une durée d'usage constante, Nous allons maintenant étudier l'impact d'une modification de ces deux paramètres (profil et durée d'usage) sur le GER du câblage et du convertisseur.

\subsection{Sensibilité à la durée d'usage}
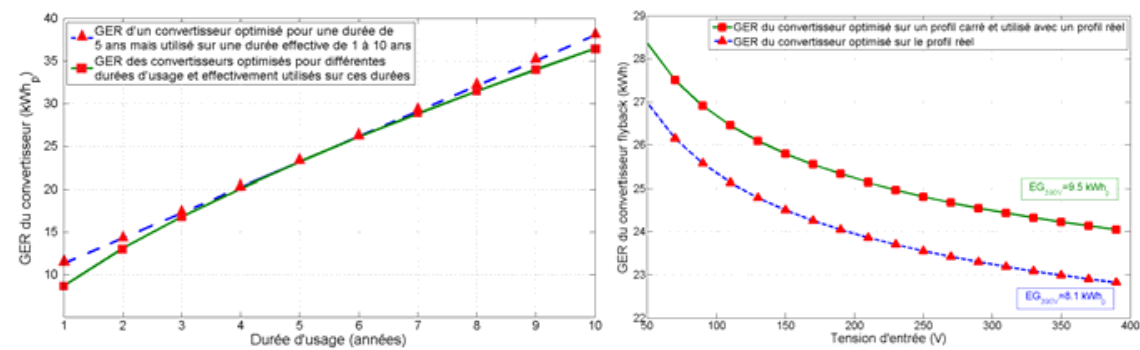

Figure 10 : Impact d'une erreur d'estimation de la durée d'usage sur le GER d'un convertisseur flyback (gauche) et Impact du choix du profil de consommation pour l'éco-dimensionnement d'un convertisseur (droite).

On présente, Figure 10 (gauche), l'évolution du GER d'un convertisseur optimisé pour une durée d'usage estimée de 5 ans et dont la durée effective d'utilisation serait différente des 5 ans estimés (courbe verte). On la compare avec la courbe représentant le GER d'un convertisseur optimisé pour la durée d'usage effective. On remarque que l'on peut commettre une erreur de $+/-40 \%$ (soit $+/-2$ ans) sur la durée d'usage servant au dimensionnement du convertisseur, sans que cela n'éloigne trop le GER du convertisseur de sa valeur optimale si le convertisseur avait été optimisé pour la durée d'usage effective.

Pour le câble, nous avions déjà montré dans (Jaouen et al 2011a) le même type de résultats concernant l'éco-dimensionnement d'un câble.

\subsection{Sensibilité au profil de consommation}

Concernant le profil de consommation, il est légitime de penser que l'optimisation serait plus simple et plus rapide dans le cas d'un profil de consommation plus simple (de type carré par exemple). Cependant il faut s'assurer que l'utilisation de ces profils pour le dimensionnement ne conduit pas à un convertisseur dont le GER serait trop éloigné de celui dimensionné en considérant le profil réel. Pour aborder cette étude, nous allons étudier l'influence du profil de consommation considéré pour éco-dimensionner un convertisseur. On présente Figure 10 l'évolution du GER, en fonction de la tension d'entrée, pour deux convertisseurs dimensionnés différemment et soumis durant leur période d'usage au profil de la Figure 3 : 
- le premier (courbe verte) a été éco-dimensionné en considérant un profil rectangulaire idéalisé correspondant à la consommation énergétique journalière et dont l'amplitude est égale à la puissance maximale consommée par la charge du profil « réel » de la Figure 3. On cherchera donc, pour le dimensionnement, à minimiser le GER du convertisseur en considérant un profil carré présentant la même énergie et la même puissance max que le profil de la Figure 3.

- le second (courbe bleue) correspond à l'éco-dimensionnement obtenu avec le profil « réel » de la Figure 3.

On remarque une différence de seulement $5 \%$ entre le GER des deux convertisseurs. L'éco-dimensionnement d'un convertisseur peut donc être réalisé sur un profil plus simple que le profil réel sur lequel il sera utilisé, pour peu que la forme simplifiée ne soit pas trop éloignée de celle du profil réel. Si l'on s'intéresse à la valeur de l'énergie grise des deux convertisseurs dans le cas d'une tension d'entrée de 390V (voir Figure 10), on remarque que le convertisseur écodimensionné sur profil carré est plus volumineux que celui éco-dimensionné sur profil réel (puisque en premier approximation, l'énergie grise est directement liée au volume). En effet, le profil carré conduit à concevoir un convertisseur ayant un rendement maximal à puissance maximale, utilisant donc des composants plus gros qu'avec profil réel, qui, dans le cas de la Figure 3, fait fonctionner le convertisseur à sa puissance maximale seulement $15 \%$ du temps.

Pour le câble, nous avions déjà montré dans (Jaouen et al 2011a) que le niveau de puissance maximale a une influence significative, à même énergie transférée, sur le GER optimal d'un câble. En effet, un profil de puissance plus impulsionnel conduit à un accroissement des pertes en fonctionnement et conduit à préférer un câble plus gros présentant un GER plus important.

\section{Conclusion}

Dans cet article, pour tenter d'apporter un nouveau regard à la question de l'intérêt de la distribution en courant continu, nous avons proposé les fondements d'une méthode de dimensionnement originale prenant en compte les indispensables convertisseurs électroniques de puissance. Le critère de dimensionnement utilisé est fondé sur l'énergie primaire consommée sur l'ensemble du cycle de vie (GER : gross energy requirement). L'objectif de cette méthode n'est alors plus de minimiser seulement les pertes en fonctionnement du système, ou sa masse, son encombrement... mais la somme de ses pertes sur sa durée d'usage et de son énergie grise.

Pour amorcer ce vaste problème et sachant qu'à notre connaissance, il n'existait pas jusqu'à présent d'éco-dimensionnement de convertisseurs statiques ni de câblage électrique, nous avons appliqué cette approche sur une portion de réseau de distribution en courant continu (envisagé pour le bâtiment), constituée d'un câble d'alimentation d'une longueur quelque peu arbitraire de $20 \mathrm{~m}$ et d'un convertisseur DC/DC flyback, le tout dimensionné sur un profil (réaliste mais également 
arbitraire) d'alimentation d'un ordinateur portable (recharge batterie et utilisation). En exploitant des documentations constructeurs et des données d'énergie grise issues d'inventaires de cycle de vie et d'autres données environnementales, nous avons établis des modèles liant les caractéristiques fondamentales des composants de puissance avec leurs dimensions afin d'établir des liens avec leurs pertes et leur énergie grise. En utilisant une approche homothétique, nous avons dimensionné ces composants afin de minimiser le GER du système considéré. L'un des objectifs étant de rechercher un niveau de tension optimal pour la distribution DC, nous avons étudié son influence sur le GER de notre système. Dans le cas considéré, avec des convertisseurs DC-DC de type flyback incluant une isolation galvanique, il parait judicieux de choisir le niveau de tension le plus élevé possible (dans les limites de la plage étudiée, jusqu'à $400 \mathrm{~V}$ ). Cependant, même si les problèmes de sécurité des personnes sont moins aigus en DC qu'en AC, les contraintes normatives de sécurité et de protection des matériels (associées à la coupure des défauts notamment) pourront amener à abaisser ce niveau de tension. De plus, d'autres études en cours montrent que, si une isolation galvanique n'est pas requise, de plus basses tensions peuvent être intéressantes.

Nous avons ensuite réalisé une étude de sensibilité portant sur l'estimation de la durée d'usage et du profil de consommation. Nous avons conclu qu'un convertisseur optimisé pour une durée d'usage de référence et utilisé sur une durée différente, dite effective, présentait finalement un GER relativement proche du GER optimal tant que l'erreur sur l'estimation de la durée d'usage ne dépassait pas $+/-40 \%$.

Rappelons que les différents composants ont été éco-dimensionnés de façon découplée selon une hypothèse de chutes de tension et de pertes relativement faibles. Dans le cas particulier de systèmes à faible durée d'usage cumulée sur le cycle de vie, il est clair que cette hypothèse risque de ne plus être respectée et il sera alors nécessaire de réaliser une optimisation globale.

Ce travail ne permet pas encore de conclure quant au niveau de tension minimisant le GER d'un réseau de distribution continu, même s'il apparaît d'ores et déjà que des tensions élevées sont préférables avec les technologies considérées. Mais il apporte une méthodologie originale de dimensionnement. Il a toutefois déjà permis précédemment (Jaouen et al 2011a, 2011b) de montrer que l'on pouvait réduire significativement la consommation en énergie des constituants (câblage et convertisseurs statiques) par rapport à ceux utilisées actuellement. La suite logique de ces travaux serait de considérer l'ensemble des constituants des convertisseurs (PCB, boîtier, radiateur, filtre CEM...) mais également d'étudier les autres types de convertisseurs électroniques présents dans une distribution en courant continu, tout particulièrement ceux de forte puissance qui se situeront à l'interface du réseau DC interne et du réseau $\mathrm{AC}$ extérieur au bâtiment qui va sans doute persister encore quelque temps. Notons également qu'une telle méthodologie permet de comparer avec des critères relativement objectifs de nouvelles technologies de composants avec de nouveaux matériaux ou concepts. Il faudrait ensuite élargir le champ d'études aux différents organes de protection. In fine, il serait possible de réaliser une comparaison entre les deux types de distribution, chacune étant constituée de 
composants éco-dimensionnés, en vue de déterminer celle présentant la plus faible consommation en énergie primaire sur cycle de vie...

Comme nous l'avons expliqué plus haut, nous avons aujourd'hui restreint nos critères de dimensionnement à la consommation en énergie primaire, il pourrait être pertinent par la suite de considérer d'autres impacts environnementaux, mais sous conditions d'arriver à obtenir les relations entre les paramètres de dimensionnement et ces impacts, informations actuellement non disponibles, au même titre que celle d'énergie primaire au départ de nos travaux. En outre, compte tenu du fait que ces éco-dimensionnements aboutissent à des systèmes nécessitant plus de matières que les systèmes actuels, le critère de consommation en ressource minérale parait être devenir significatif.

Enfin, précisons que cette méthodologie d'éco-dimensionnement n'est pas exclusivement dédiée à l'étude de réseaux de distribution mais qu'elle peut être étendue à tout type de système énergétique électrique.

Tableau 1. Tableau récapitulatif des modèles développés pour cette étude et des paramètres imposés

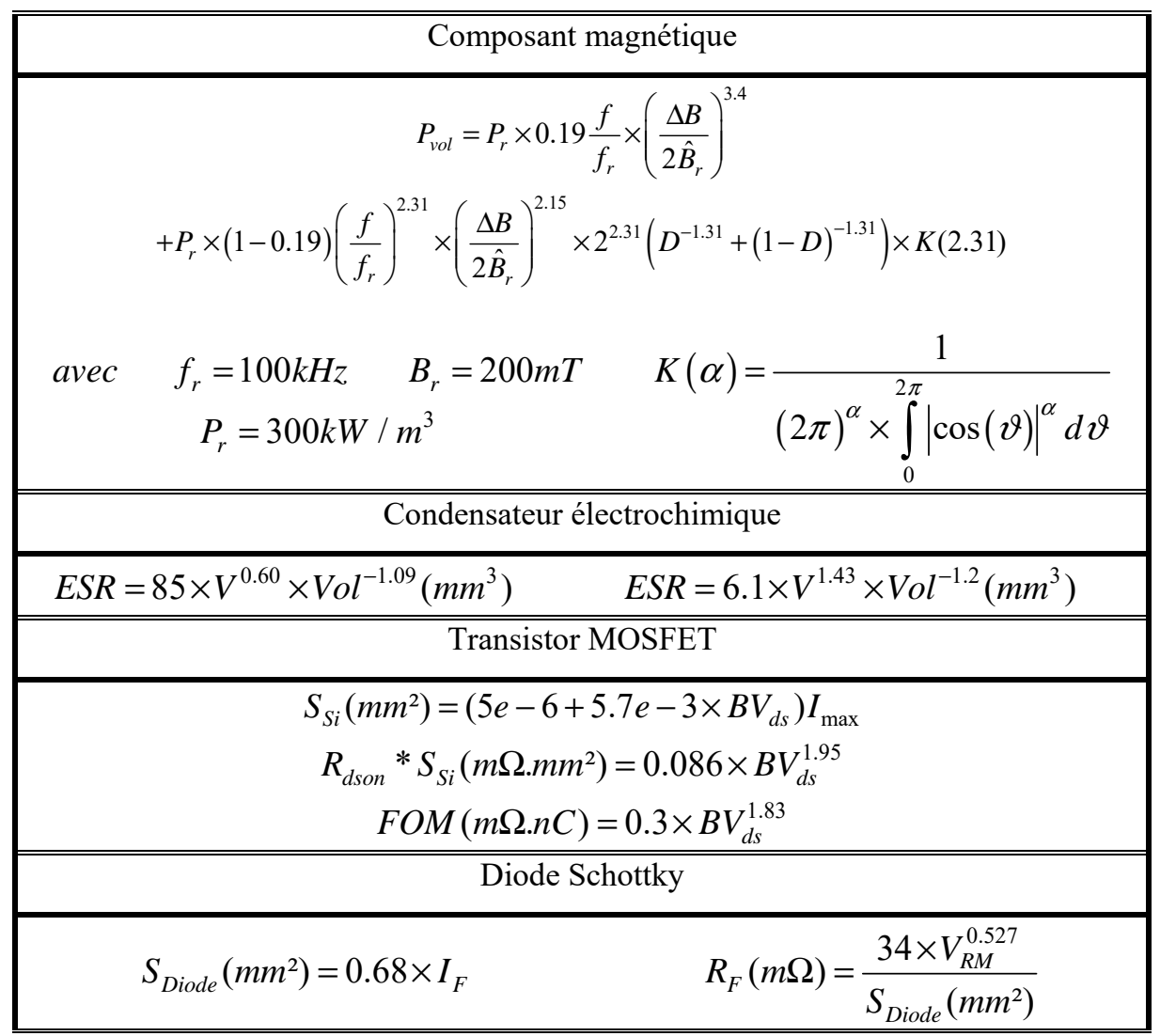




\section{Références}

Boughamni W., Roger D., Manata J.P., Brudny J.F, Frezel PH., (2010). « Analyse comparative de l'imprégnation d'un bobinage et de diverses solutions de Thermo collage ", CONFREGE, Toulouse 2010, 7p.

Brush L., (2012). « Data Centers Drive Killer Power Apps », Bodo’s Power Systems, April 2012, pp.20-21.

Chalmers university (2000). Capacitor for hole mounting assembly, Donnée ACV extraite de la base de donnée de l'université de Chalmers, Mars 2000, visité en Dec 2011 (http://www.cpm.chalmers.se/CPMDatabase/Scripts/sheet.asp?ActId=ECOP3207)

Debusschere V., (2009). Contributions méthodologiques à l'éco-conception de convertisseurs électromagnétiques d'énergie, thèse en Electronique, Electrotechinque et Automatique de l'ENS de Cachan, soutenue le 9 décembre 2009.

Dones R. et al., (2007). Life Cycle Inventories of Energy Systems : Results for Current Systems in Switzerland and other UCTE Countries, rapport Ecoinvent n ${ }^{\circ} 2007$.

EIME (2009). Base de données EIME V11.0, site web EIME, visité en Déc 2010 (http://www.codde.fr/page.php?rubrique=20)

Ejury J., (2003). How to compare the Figure Of Merite (FOM) of MOSFETs, Application note Infineon, Juin 2003.

Engelen K, et al, (2006). "The Feasibility of Small-Scale Residential DC Distribution Systems », IEEE Industrial Electronics Society, CNAM Paris, France.

Hadj Amor A., Poloujadoff M., Salon S. J., (1998). «Optimum design of electromagnetic devices by the state variable method: application to transformers ", Optimization of Electrical and Electronic Equipment, OPTIM '98, May 98.

Hischier R., (2007). Life cycle inventories of packaging \& graphical papers, part 2: Plastics, Rapport Ecoinvent $n^{\circ} 11$, Dec. 07

Hischier R., Classen M., Lehmann M., Scharnhorst W., (2007). Life cycle inventories of electric and electronic equipment: Product use and disposal Part III \$5, Rapport Eco-invent $\mathrm{n}^{\circ} 18$, Dec 2007.

Jaouen C., Multon B., Barruel F., (2011). «Wiring design based on Global Energy Requirement criteria: a first step towards optimization of DC distribution voltage ", iREED'11, Lille, France.

Jaouen C., Multon B., Barruel F., (2011). « Design optimization methodology for power converters based on global energy requirement criteria. Application to a DC-DC flyback structure », European conference on Power Electronics, EPE'11, Birmingham, Aug 2011

John Shen Z., Xiong Y., Cheng X., Fu Y., Kumar P., (2006). « Power MOSFET switching loss analysis: a new insight », Conference Record of the 2006 IEEE Industry Applications Conference, Tampa, Finland, Oct 2006, 5p.

Meyer J.P., Poyard J.L,. (2006). «Prévention des risques professionnels, risques liés aux installations », Techniques de l'Ingénieur, SE 3850. 
Multon B., Ben Ahmed H., Debusschere V., Thiaux Y., Aubry J., Jaouen C., Barruel F., (2010). « Expériences de recherche en éco-conception dans le domaine du Génie Electrique », CONFREGE, Toulouse, déc. 2010, 8p.

NF (1998). « Norme NF C 32-201, Conducteurs et câbles isolés au polychlorure de vinyle de tension assignée au plus égale à 450/750V », 1998, Révisé en 2009

Sannino A., Postaglione G., et Bollen H.J., (2003). «Feasibility of a DC network for commercial facilities », IEEE Transactions on Industry applications, vol. 39, no. 5, oct. 2003.

Sechilariu M., (2007). «C.A vs C.C : Intégrer un réseau électrique hybride dans les bâtiments équipés en énergies renouvelables », EF 2007, Toulouse, 2007.

Tikana L., Sievers H., Klassert A., (2005). Life cycle assessment of copper product, European Copper Institute.

Van den Bossche A.P., Van de Sype D.M., Valchev V.C, (2005). « Ferrite loss measurement and models in half bridge and full bridge waveforms ", IEEE 36th Power Electronics Specialists Conference PESC'05, Recife, Brazil, 8p.

Vishay (2010). Documentation technique de condensateurs électrochimiques (séries 058/059 PLL-SI), Site web de la société Vishay (http://www.vishay.com), visité en Nov 2010.

Vishay (2010) Documentation technique de puces de diodes Schottky haute performance fabriquées par Vishay, site web de Vishay, visité en Nov. 2010 (http://www.vishay.com/diewafer/schottky_die/). 